\title{
MEASURABLE REALIZATIONS OF ABSTRACT SYSTEMS OF CONGRUENCES
}

\author{
CLINTON T. CONLEY ${ }^{1}$, ANDREW S. MARKS $^{2}$ and SPENCER T. UNGER ${ }^{3}$ \\ ${ }^{1}$ Department of Mathematical Sciences, Carnegie Mellon University, Pittsburgh, PA 15213, USA; \\ email: clintonc@andrew.cmu.edu \\ ${ }^{2}$ Department of Mathematics, University of California at Los Angeles, USA; \\ email: marks@math.ucla.edu \\ ${ }^{3}$ Department of Mathematics, Hebrew University of Jerusalem, Israel; \\ email: unger.spencer@mail.huji.ac.il
}

Received 14 May 2019; accepted 15 January 2020

\begin{abstract}
An abstract system of congruences describes a way of partitioning a space into finitely many pieces satisfying certain congruence relations. Examples of abstract systems of congruences include paradoxical decompositions and $n$-divisibility of actions. We consider the general question of when there are realizations of abstract systems of congruences satisfying various measurability constraints. We completely characterize which abstract systems of congruences can be realized by nonmeager Baire measurable pieces of the sphere under the action of rotations on the 2-sphere. This answers a question by Wagon. We also construct Borel realizations of abstract systems of congruences for the action of $\mathrm{PSL}_{2}(\mathbb{Z})$ on $\mathrm{P}^{1}(\mathbb{R})$. The combinatorial underpinnings of our proof are certain types of decomposition of Borel graphs into paths. We also use these decompositions to obtain some results about measurable unfriendly colorings.
\end{abstract}

2010 Mathematics Subject Classification: 03E15 (primary); 28A75, 37A20, 52B45 (secondary)

\section{Introduction}

Recently, several results have been proved about the extent to which realizations of geometrical paradoxes can be found with sets having measurability properties such as being Borel, Lebesgue measurable, or Baire measurable (see for instance [CS, DF, GMP16, GMP17, Ma, MU16, MU17]). This is a growing area of study at the interface of descriptive set theory, combinatorics, and ergodic theory. This paper is a contribution to this study. One of the earliest results in

(C) The Author(s) 2020. This is an Open Access article, distributed under the terms of the Creative Commons Attribution licence (http://creativecommons.org/licenses/by/4.0/), which permits unrestricted re-use, distribution, and reproduction in any medium, provided the original work is properly cited. 
this vein is the theorem of Dougherty and Foreman [DF] that the Banach-Tarski paradox can be realized using Baire measurable pieces. In contrast to the classical Banach-Tarski paradox, which uses five pieces, Dougherty and Foreman's Baire measurable solution uses six pieces. A result of Wehrung [Weh] implies that this is optimal; there is no Baire measurable realization of the Banach-Tarski paradox with five pieces. This suggests a subtle difference between the classical and Baire measurable contexts.

In this paper, we consider a refined framework called 'abstract systems of congruence' for describing when an action can be partitioned into finitely many pieces satisfying certain congruence relations. As one application, we give an exact characterization of which abstract systems of congruences can be realized in the 2-sphere with arbitrary pieces versus nonmeager Baire measurable pieces. This refines the dual results of Wehrung [Weh], and Dougherty and Foreman [DF].

We formally define abstract systems of congruences as follows. Given a set $S$, its proper powerset $\mathcal{P}_{\mathrm{pr}}(S)$ is $\mathcal{P}_{\mathrm{pr}}(S)=\{R \subseteq S: R \neq \emptyset \wedge R \neq S\}$. Following Wagon [W, Definition 4.10], an abstract system of congruences on $n=\{0, \ldots$, $n-1\}$ is an equivalence relation $E$ on $\mathcal{P}_{\mathrm{pr}}(n)$ so that if $U E V$, then $U^{\mathrm{c}} E V^{\mathrm{c}}$. Here $U^{\text {c }}$ denotes the complement of $U$. Suppose $a: \Gamma \curvearrowright X$ is an action of a group on a set $X$. Then we say that $A, B \subseteq X$ are $a$-congruent if there is a group element $\gamma \in \Gamma$ such that $\gamma \cdot A=B$. An a-realization of an abstract system of congruences $E$ is a partition $\left\{A_{0}, \ldots, A_{n-1}\right\}$ of $X$ such that for all $U, V \in \mathcal{P}_{\text {pr }}(n)$ with $U E V$, we have that $\bigcup_{i \in U} A_{i}$ and $\bigcup_{i \in V} A_{i}$ are $a$-congruent. The definition of an abstract system of congruences reflects the fact that congruence is an equivalence relation and that if $A, B \subseteq X$ are congruent, then $A^{\mathrm{c}}$ and $B^{\mathrm{c}}$ are also congruent.

An important example of an abstract system of congruences is the smallest abstract system of congruences $E$ on $\mathcal{P}_{\mathrm{pr}}(4)$ containing the relations $\{0\} E\{0$, $1,2\}$ and $\{1\} E\{0,1,3\}$. A realization of this system gives a paradoxical decomposition since $\{0,3\}$ and $\{1,2\}$ partition $\{0,1,2,3\}$. The translation action of the free group on two generators $\mathbb{F}_{2}$ on itself is an example of an action realizing this system of congruences [W, Theorem 4.2]. Another important example of an abstract system of congruences is the smallest abstract system of congruences $E$ on $\mathcal{P}_{\mathrm{pr}}(n)$ where $\{i\} E\{j\}$ for every $i, j \in n$. An action is said to be $n$-divisible if it satisfies this system of congruences (that is, it can be partitioned into $n$ congruent pieces). For example, it is easy to see that the action of the rotation group $\mathrm{SO}_{3}$ on the 2-sphere is not 2-divisible by considering the 'poles' of the rotation. However, this action is $n$-divisible for $n \geqslant 3$ (see [W, Corollary 4.14]).

Wagon has characterized which abstract systems of congruences can be realized in the action of the group $\mathrm{SO}_{3}$ of rotations on the 2-sphere. We say that an abstract 
system of congruences $E$ on $n$ is noncomplementing if there is no set $X \in \mathcal{P}_{\mathrm{pr}}(n)$ such that $X E X^{\mathrm{c}}$.

THEOREM 1.1 [W, Corollary 4.12]. Suppose $E$ is an abstract system of congruences. $E$ can be realized in the action of $\mathrm{SO}_{3}$ on the 2-sphere if and only if $E$ is noncomplementing.

We show that in order to realize an abstract system of congruences with Baire measurable pieces in the sphere, we need one additional property. We say that an abstract system of congruences $E$ on $\mathcal{P}_{\mathrm{pr}}(n)$ is nonexpanding if there do not exist sequences of sets $\left(V_{i}\right)_{i \leqslant k}$ and $\left(W_{i}\right)_{i \leqslant k}$, where $V_{i} E W_{i}$ for every $i \leqslant k$ and $W_{i} \subseteq V_{i+1}$ for every $i<k$, such that $W_{k} \subsetneq V_{0}$. Hence,

$$
V_{0} E W_{0} \subseteq V_{1} E W_{1} \subseteq \cdots V_{k} E W_{k} \subsetneq V_{0}
$$

THEOREM 1.2. Suppose $E$ is an abstract system of congruences. Then $E$ can be realized in the action of $\mathrm{SO}_{3}$ on the 2-sphere with Baire measurable pieces, each of which is nonmeager, if and only if $E$ is noncomplementing and nonexpanding.

This theorem positively answers Wagon's question [W, page 47] of whether the 2 -sphere is $n$-divisible with Baire measurable pieces for $n \geqslant 3$. Indeed, the smallest abstract system of congruences $E$ containing the relations $\{1\} E\{2\}$ $E \cdots E\{n\}$ is clearly noncomplementing and nonexpanding for $n \geqslant 3$, and hence has a Baire measurable realization in the action of $\mathrm{SO}_{3}$ on the 2-sphere. Wagon has also asked whether the 2-sphere is $n$-divisible into Lebesgue measurable pieces. This remains an open problem.

Let $\mathbb{F}_{n}$ be the free group on $n$ generators. Our proof of Theorem 1.2 shows more generally that if $n \geqslant 2$, then any free Borel action of $\mathbb{F}_{n}$ on a Polish space $X$ can realize an abstract system of congruences that is nonexpanding and noncomplementing using Baire measurable pieces (see Lemma 3.6).

Our main tool for proving Theorem 1.2 is a decomposition lemma for acyclic Borel graphs into sets of paths with a property concerning how the paths from different sets may overlap.

Definition 1.3. Suppose $G$ is a graph and $G_{0}, G_{1}, \ldots$ is a sequence of subgraphs of $G$. Then we say $G_{0}, G_{1}, \ldots$ is end-ordered if for all vertices $x$ in $G$, if $x$ is a vertex in $G_{i}$ and $G_{j}$ where $i<j$, then $x$ is a leaf in $G_{j}$. Similarly, if $S_{0}, S_{1}, \ldots$ are sets of subgraphs of $G$, then we say that $S_{0}, S_{1}, \ldots$ is end-ordered if for all vertices $x$ in $G$, if $x$ is a vertex in $H \in S_{i}$ and a vertex in $H^{\prime} \in S_{j}$ where $i<j$, then $x$ is a leaf in $H^{\prime}$. 
Definition 1.4. Suppose $G$ is an acyclic Borel graph. Then a path decomposition of $G$ is a sequence $P_{0}, P_{1}, \ldots$ of sets of paths in $G$ such that $P_{0}, P_{1}, \ldots$ is end-ordered, every $P_{i}$ consists of vertex-disjoint paths, and for every edge $e$ in $G$, there exists exactly one $P_{i}$ so that $e$ appears in a path in $P_{i}$. We say that a path decomposition is Borel if each set $P_{i}$ is Borel, and the path decomposition has length at least $n$ if every path has length at least $n$.

Roughly, a path decomposition is a way of covering the graph with sets of paths $P_{0}, P_{1}, \ldots$ so that all the paths in $P_{j}$ have interiors that are disjoint from the paths in $P_{i}$, for $i<j$.

One of our main lemmas (Lemma 3.4) says that if $G$ is a locally finite acyclic Borel graph, then for all $n$, there is a comeager set on which $G$ has a Borel path decomposition of length at least $n$.

A different case in which we have Borel path decompositions is when we have Borel end selections. Recall that if $G$ is a graph on $X$, a ray is an infinite simple path in $G$, and that two rays $\left(x_{i}\right)_{i \in \mathbb{N}}$ and $\left(y_{i}\right)_{i \in \mathbb{N}}$ are end-equivalent if for every finite set $S \subseteq X$, the rays $\left(x_{i}\right)$ and $\left(y_{i}\right)$ eventually lie in the same connected component of $G\lceil(X \backslash S)$. An end of $G$ is an end-equivalence class of $G$. If $G$ is a Borel graph on $X$, we say that $G$ admits a Borel selection of finitely $k$ ends in each connected component if there are Borel functions $r_{0}, \ldots, r_{k-1}$ sending each $x \in X$ to $k$ end-inequivalent rays $r_{0}(x), \ldots, r_{k}(x)$ in the connected component of $x$ such that if $y$ are in the same connected component of $G$ as $x$, then $\left\{r_{0}(x), \ldots, r_{k-1}(x)\right\}$ and $\left\{r_{0}(y), \ldots, r_{k-1}(y)\right\}$ are representatives of the same set of ends. We say that $G$ admits a Borel selection of finitely many ends in each connected component if $G$ can be partitioned into countably many invariant Borel sets $A_{0}, A_{1}, \ldots$ so that for each $i$, there is some $k$ so that $G \uparrow A_{i}$ has a Borel selection of $k$ ends in each connected component.

We show that if $G$ is an acyclic bounded degree Borel graph on $X$ such that there is a Borel selection of finitely many ends in every connected component of $G$, then for every $n$ we can find a Borel path decomposition of $G$ of length at least $n$ (see Lemma 4.2). We construct explicit realizations of abstract systems of congruences for the action of $\mathrm{PSL}_{2}(\mathbb{Z})$ on $P^{1}(\mathbb{R})$ by combining this lemma with an explicit end selection defined using continued fraction expansions.

THEOREM 1.5. Suppose $E$ is an abstract system of congruences, which is noncomplementing and nonexpanding. Then $E$ can be realized in the action of $\mathrm{PSL}_{2}(\mathbb{Z})$ on $P^{1}(\mathbb{R})$ by Borel pieces.

For example, this action is $n$-divisible using Borel pieces for $n \geqslant 3$. 
OPEN PROBLEM 1.6. Characterize the abstract systems of congruences, which can be realized in the action of $\mathrm{PSL}_{2}(\mathbb{Z})$ on $P^{1}(\mathbb{R})$ by Borel pieces.

It is a theorem of Adams [JKL, Lemma 3.21] that if $G$ is a locally finite graph on a standard probability space $(X, \mu)$ and $G$ is $\mu$-hyperfinite, then $G$ admits a $\mu$-measurable selection of finitely many ends. Using the Adams theorem, we also show that any $\mu$-hyperfinite action of $\mathbb{F}_{2}$ on a standard probability space $(X, \mu)$ has a $\mu$-measurable realization of any abstract system of congruences $E$ if $E$ is noncomplementing and nonexpanding (see Theorem 4.3).

Our decomposition lemmas have some other applications in Borel combinatorics. Simon Thomas has asked whether every locally finite Borel graph has an unfriendly Borel coloring, where an unfriendly coloring of a graph $G$ on $X$ is a function $f: X \rightarrow 2$ such that for every $x$,

$$
|\{y \in N(x): c(x) \neq c(y)\}| \geqslant|\{y \in N(x): c(x)=c(y)\}| .
$$

The question by Thomas is partially motivated by the open problem in classical combinatorics of whether every countable graph admits an unfriendly coloring. If $G$ is a graph on $X$, we say that a function $f: X \rightarrow 2$ is strongly unfriendly if for every $x, \mid\{y \in N(x): c(x)=c(y)\} \leqslant 1$.

We use our decomposition lemma to prove the following result.

THEOREM 1.7. Suppose $G$ is a locally finite acyclic Borel graph on a Polish space $X$ that admits a Borel path decomposition of length at least 5. Then $G$ has a Borel strongly unfriendly coloring. Hence, if $G$ is a locally finite acyclic Borel graph of degree at least 2, then $G$ admits a Baire measurable strongly unfriendly coloring, and $G$ admits a $\mu$-measurable strongly unfriendly coloring for every Borel probability measure on $X$ rendering $G \mu$-hyperfinite.

In Section 6, we also discuss some further applications of our decompositions, such as new proofs of Baire measurable and $\mu$-measurable edge coloring and matchings.

\section{Preliminaries}

Our notation for graph theory is standard; see [D]. We recall a few notions. By a graph on $X$, we mean a simple undirected graph with vertex set $X$. The degree of a vertex is its number of neighbors. Two vertices are adjacent if there is an edge between them. A vertex is a leaf if it has degree 1, and is a splitting vertex if it has degree at least 3 . By a path, we mean a simple path of finite length $x_{0}, \ldots, x_{n}$. 
The endpoints of the path are $x_{0}$ and $x_{n}$, and the remaining vertices are interior vertices of the path. By a ray, we mean a simple infinite path $\left(x_{i}\right)_{i \in \mathbb{N}}$.

If $G$ is a graph, we say that a set of vertices is independent if it does not contain two adjacent vertices. We say that a set $A$ is $k$-independent if for all distinct $x$, $y \in A$, we have $d(x, y)>k$.

Suppose $G$ is a graph on $X$. A subgraph $H$ of $G$ is a graph on a subset of $X$ so that every edge in $H$ is an edge in $G$. If $Y \subseteq X$, the restriction of $G$ to $Y$ or induced subgraph on $Y$, denoted by $G \uparrow Y$, is the graph on $Y$ where the edges of $G \uparrow Y$ are all edges in $G$ with vertices in $Y$.

A Borel graph is a graph on a Polish space $X$ whose edge relation is Borel. For background on Borel graphs, see [KM]. An important example of a Borel graph arises from Borel group actions. If $a$ is a Borel action of a countable group $\Gamma$ on a Polish space $X$ and $S \subseteq \Gamma$ is a symmetric set of group elements, then we let $G(a, S)$ be the graph on $X$, where $x, y$ are adjacent, if there exists some $\gamma \in S$ such that $\gamma \cdot x=y$.

If $G$ is a Borel graph on $X$, the set of all paths of $G$ is a Borel subset of $\bigcup_{n} X^{n}$, and hence a standard Borel space. Hence, we may speak about a set of paths in $G$ being Borel.

We note that in contrast to Lemmas 3.4 and 4.2, there exist Borel graphs that do not admit Borel path decompositions of length at least 3.

THEOREM 2.1. Suppose that $G$ is a Borel graph of degree at least 3 on a Polish space $X$ that admits an invariant measure $\mu$. Then $G$ does not admit a Borel path decomposition on any $\mu$-conull Borel set.

Proof. Let $P_{0}, P_{1}, \ldots$ be a Borel path decomposition. Note that every vertex $x$ must be the endpoint vertex of some unique path $p(x) \in P_{i}$ since $G$ has degree at least 3. Define a Borel function $f: X \rightarrow X$, where $f(x)$ is the vertex adjacent to $x$ in $p(x)$. Then $f$ is a compression function contradicting $\mu$ being measurepreserving $[\mathbf{N}]$.

We will use the following lemma giving a criterion for the existence of abstract systems of congruences without any measurability properties.

Definition 2.2. Suppose $E$ is an abstract system of congruences. We say that a relation $R$ on a set $X$ generates the equivalence relation $E$ on $X$ if the smallest abstract system of congruences containing $R$ is equal to $E$. We say that a generating set $R$ for $E$ is good if $R$ contains all pairs $(U, V) \in E$ such that $U=V^{\mathrm{c}}$. Finally, a minimal good generating set of $E$ is a good generating set $R$ so that there is no proper subset of $R$ that is a good generating set for $E$. 
Lemma 2.3 (See also [W, Section 4]). Suppose that $E$ is an abstract system of congruences on $n$, and $R=\left\{\left(S_{1}, T_{1}\right), \ldots,\left(S_{k}, T_{k}\right)\right\}$ is a minimal good generating set of E. Suppose $a: \Gamma \curvearrowright X$ is an action of

$$
\Gamma=\left\langle\gamma_{1} \cdots \gamma_{k} \mid\left\{\gamma_{i}^{2}=1: T_{i}=S_{i}^{c}\right\}\right\rangle \text {. }
$$

Suppose finally that for every $x \in X, \operatorname{Stab}(\{x\})$ is cyclic. Then there is an arealization $\left\{A_{0}, \ldots, A_{n-1}\right\}$ of $E$, witnessed by

$$
\gamma_{i} \cdot \bigcup_{j \in S_{i}} A_{j}=\bigcup_{j \in T_{i}} A_{j} .
$$

Proof. This lemma is proved in [W, Section 4] when $E$ is noncomplementing.

Using the axiom of choice, it suffices to prove the lemma when the action has a single orbit. Since the stabilizer of every point is cyclic, the graph $G\left(a,\left\{\gamma_{i}: i \leqslant\right.\right.$ $k\})$ has at most one cycle.

Suppose there is a cycle $x_{0}, x_{1}, \ldots, x_{l}=x_{0}$. Let $g$ be the group element $g=g_{l-1} \cdots g_{1} g_{0}$ so that $x_{i+1}=g_{i} \cdots g_{1} g_{0} \cdot x_{0}$, and $g_{i} \in\left\{\gamma_{1}^{ \pm}, \ldots, \gamma_{k}^{ \pm}\right\}$. We claim we can assign elements of this cycle to $A_{0}, \ldots, A_{n-1}$ in a way that is consistent with (*). First, define functions $X$ and $Y$ on the generators by letting $X\left(\gamma_{j}\right)=S_{j}$, $Y\left(\gamma_{j}\right)=T_{j}, X\left(\gamma^{-1}\right)=T_{j}$, and $Y\left(\gamma^{-1}\right)=S_{j}$; so obeying (*) corresponds to having

$$
x_{i} \in \bigcup_{j \in X\left(g_{i}\right)} A_{j} \text { iff } x_{i+1} \in \bigcup_{j \in Y\left(g_{i}\right)} A_{j} .
$$

Let $i^{+}$denote $i+1 \bmod k$.

Case 1: Suppose there is some $i<l$ such that $X\left(g_{i^{+}}\right) \neq Y\left(g_{i}\right)$ and $X\left(g_{i^{+}}\right) \neq$ $Y\left(g_{i}\right)^{\mathrm{c}}$. Then we claim we can assign $x_{0}, \ldots, x_{l}$ to $A_{0}, \ldots, A_{n-1}$ in a way that satisfies (*). For example, suppose there is $r, s \in X\left(g_{i^{+}}\right)$such that $r \in Y\left(g_{i}\right)$ and $s \in Y\left(g_{i}\right)^{\mathrm{c}}$. Start by assigning $x_{i^{++}}$to an arbitrary element of $Y\left(g_{i^{+}}\right)$. Then proceed around the cycle, assigning elements in a way consistent with (*). Finish by assigning $x_{i^{+}}$to $A_{r}$ if $x_{i} \in X\left(g_{i}\right)$, or assigning $x_{i^{+}}$to $A_{s}$ if $x_{i} \notin X\left(g_{i}\right)$. The other cases are essentially identical.

Case 2: Suppose for all $i<l, X\left(g_{i^{+}}\right)=Y\left(g_{i}\right)$ or $X\left(g_{i^{+}}\right)=Y\left(g_{i}\right)^{\mathrm{c}}$. In this case, we claim that if there is no way to assign $x_{0}, \ldots, x_{l}$ to $A_{0}, \ldots, A_{n-1}$ in a way that satisfies $\left(^{*}\right)$, then $R$ is not a minimal good generating set, which is a contradiction.

Let $V(0)=X\left(g_{0}\right)$, and then inductively define $V(i+1)=Y\left(g_{i}\right)$ if $V(i)=$ $X\left(g_{i}\right)$, and otherwise $V(i+1)=V\left(g_{i}\right)^{\mathrm{c}}$ if $V(i)=X\left(g_{i}\right)^{\mathrm{c}}$. Hence, $V(0) E V(1) E$ $V(2) \cdots E V(l)$. Since there is no way to assign $x_{0}, \ldots, x_{l}$ to $A_{0}, \ldots, A_{n-1}$ in a way that satisfies $\left(^{*}\right)$, we must have that $V(0)=V(l)^{\mathrm{c}}$. Now take a minimal length 
subsequence $V(i), \ldots, V(j)$ of $V(0), \ldots, V(l)$ such that

$$
j-i \geqslant 2 \text { and } V(i)=V(j) \text { or } V(i)=V(j)^{\mathrm{c}} .
$$

It is clear that if $g_{i}=\gamma_{m}$, then we can remove the pair $\left(S_{m}, T_{m}\right)$ from $R$ and we would still generate $E$. This is because if $V(i)=V(j)$, then $V(i) E V(i+1)$ follows from $V(i+1) E V(i+2) \cdots E V(j)=V(i)$. If $V(i)=V(j)^{\mathrm{c}}$, then the fact that $V(i) E V(i+1)$ follows from $V(i+1) E V(i+2) \cdots E V(j)=V(i)^{\mathrm{c}}$, and by the definition of a good generating set, the pair $V(j) E V(i)^{\mathrm{c}}$ must appear in $R$. (Note that here we are using the minimal length of this subsequence among those with (**) and the fact that $g$ is a reduced word to ensure that the equivalences $V(i) E V(i+1)$ and $V(i) E V(i+1)^{\mathrm{c}}$ do not appear in the equivalences $V(i+1) E \cdots E V(j))$. This finishes Case 2.

Now that we assigned the elements of the cycle to $A_{0}, \ldots, A_{n-1}$, if a cycle exists, for the remaining acyclic portion of the graph, we clearly iteratively assign the vertices to $A_{0}, \ldots, A_{n-1}$ in a way that satisfies (*).

Throughout, we will be working with actions of such groups $\Gamma$ that are free products of copies of $\mathbb{Z}$ and $\mathbb{Z} / 2 \mathbb{Z}$ and where the generators of $\Gamma$ of order 2 will witness congruences of the form $U E U^{\mathrm{c}}$.

\section{Baire measurable realizations}

In this section, we prove Theorem 1.2. We begin with a decomposition lemma for acyclic locally finite Borel graphs (Lemma 3.4). As an intermediate step toward this lemma, we consider decompositions into certain types of trees that themselves have suitable decompositions into paths. Recall that a tree is a connected acyclic graph, a leaf of a tree is a vertex of degree 1, and a splitting vertex is a vertex of degree at least 3 . We say that a tree $T$ is $n$-spindly if there is at most one leaf $l$ of $T$ so that for all distinct leaves $x, y$, if $l \notin\{x, y\}$, then $d(x, y)>2 n$, and if $l \in\{x, y\}$, then $d(x, y) \geqslant n$. The utility of spindly trees is given by the following lemma.

LEMMA 3.1. Every finite $n$-spindly tree $T$ can be written as a union of edgedisjoint paths $p_{0}, p_{1}, \ldots$ each having length at least $n$, and which are endordered.

Proof. We construct $p_{0}, p_{1}, \ldots, p_{k}$ by induction. Let $p_{0}$ be a path from one leaf to another leaf, having minimal length among such paths between leaves. Let the endpoints of $p_{0}$ be $x$ and $y$. We may assume $x \neq l$ for the distinguished leaf $l$ if it exists. 
For each vertex $z$ not in $p_{0}$, let $V_{z}$ be the set of $w$ such that there is a path $p$ from $z$ to $w$ such that no interior vertex of $p$ is in $p_{0}$. Since $T$ is a tree, there is exactly one vertex in $T \nmid V_{z}$, which is contained in $p_{0}$. Let this vertex be $l_{z}$, which is a leaf in $T \uparrow V_{z}$. For any leaf $w$ in $T \uparrow V_{z}$, the distance $d\left(x, l_{z}\right) \leqslant d\left(w, l_{z}\right)$. Otherwise if $d\left(w, l_{z}\right)<d\left(x, l_{z}\right)$, the path from $w$ to $y$ would have smaller length than $p_{0}$, but $p_{0}$ has minimal length. Hence, $d\left(w, l_{z}\right) \geqslant n$ since otherwise $d\left(x, l_{z}\right) \leqslant$ $d\left(w, l_{z}\right)<n$, which implies that $d(x, w)<2 n$, contradicting $T$ being $n$-spindly since neither $x$ nor $w$ is equal to $l$. It follows that $T \uparrow V_{z}$ is $n$-spindly, witnessed by $l_{z}$.

The lemma follows by inductively applying the lemma to all these $n$-spindly subgraphs of the form $T \uparrow V_{y}$.

REMARK 3.2. Every locally finite $n$-spindly tree $T$ can be written as a union of edge-disjoint paths $p_{0}, p_{1}, \ldots$ that are of length at least $n$ and which are endordered. That is, Lemma 3.1 remains true for infinite $n$-spindly trees. This is by an infinite iteration of the same process in the proof of Lemma 3.1 (or by a compactness argument).

As an intermediate step toward our path decomposition, we prove a lemma decomposing into $n$-spindly trees.

Lemma 3.3. Suppose $G$ is a locally finite acyclic graph on a Polish space $X$ of degree at least 2 , and $n \geqslant 1$. Then there are a $G$-invariant comeager Borel set $D$ and edge-disjoint Borel subgraphs $G_{0}, G_{1}, \ldots$ such that $\bigcup_{i} G_{i}=G \uparrow D$, every connected component of $G_{i}$ is a finite n-spindly tree, and the sequence $G_{0}, G_{1}, \ldots$ is end-ordered.

Proof. We give a construction in countably many steps. Let $d(i)=3 n 6^{i}$. By [MU16, Lemma 3.1], let $\left(A_{i}\right)_{i \in \mathbb{N}}$ be subsets of $X$ such that the elements of $A_{i}$ are pairwise of distance greater than $3 d(i)$, and $D=\bigcup_{i} A_{i}$ is comeager and $G$-invariant. Before step $s$, we will have constructed edge-disjoint Borel subgraphs $G_{0}, G_{1}, \ldots, G_{s-1}$. Let $H_{i}=\bigcup_{j \leqslant i} G_{j}$. Let $H_{i, k}$ for $k \leqslant i$ be all the connected components $C$ in $H_{i}$, where $k$ is least such that $C$ is also a connected component of $H_{k}$. So $H_{i}$ is the disjoint union $H_{i}=\bigcup_{k \leqslant i} H_{i, k}$.

Our induction hypotheses are as follows:

(1) For every $i<s$ and $x \in A_{i}$, there is an edge incident to $x$ in $H_{i}$.

(2) For every $k \leqslant s-1$, the diameter of any connected component of $H_{s-1, k}$ is at most $d(k)$. 
(3) For every $k \leqslant s-1$, the distance between any two connected components of $H_{s-1, k}$ is at least $2 d(k)$.

(4) The distance between any two connected components of $H_{s-1}$ is greater than $2 n$.

Note that these hypotheses imply that every edge in $G \uparrow D$ will appear in some $G_{i}$. To see this, suppose $x, x^{\prime}$ are adjacent, where $x \in A_{s}$ and $x^{\prime} \in A_{s^{\prime}}$. Then $x$ and $x^{\prime}$ must both be in connected components of $H_{\max \left(s, s^{\prime}\right)}$ by (1), and hence the same connected component by (4). Thus the edge $\left(x, x^{\prime}\right)$ must be in $H_{\max \left(s, s^{\prime}\right)}$ since $G$ is acyclic.

Below we inductively define $G_{s}$ and then prove that each connected component of $G_{s}$ is $n$-spindly. Note that to satisfy part (1) of the induction hypothesis, we need to add an edge incident to each $x \in A_{s}$ to $G_{s}$ if there is not one already in $H_{s-1}$. However, simply adding such edges by themselves may violate induction hypothesis (4). So we will need to inductively define $G_{s}$ to include paths to all nearby connected components of $H_{s-1, k}$ so that they all become the same connected component in $H_{s}$. Hypotheses (2) and (3) give us control over this process, so we can satisfy (4).

To begin, let $G_{s, 0}$ be the graph consisting of all vertices in $A_{s}$ (and no edges). Inductively, for $0<i \leqslant s$, let $G_{s, i} \supseteq G_{s, i-1}$ be the union of $G_{s, i-1}$ with all paths of length at most $d(s-i)$ in the graph $G \backslash H_{s-1}$ from vertices in $G_{s, i-1}$ to connected components of $H_{s-1, s-i}$. Since elements of $A_{s}$ have pairwise distance at least $3 d(s)$, it is clear by induction that components of $G_{s, i}$ have diameter at most $2 d(s-1)+\cdots+2 d(s-i)$, and hence components of $G_{s, s}$ have diameter at most $2 d(s-1)+\cdots+2 d(0)$. Similarly, the components of $G_{s, s}$ are pairwise of distance at least $3 d(s)-2 d(s-1)-\cdots-2 d(0)$.

Let $A_{s}^{0}$ be the set of $x \in A_{s}$ that are not incident to any edge of $H_{s-1}$ or $G_{s, s}$. (Hence, every $x \in A_{s}^{0}$ has $d\left(x, H_{s-1}\right)>d(0) \geqslant 3 n$.) For each $x \in A_{s}^{0}$, let $p(x)$ be the lex-least path of length $n$ in $G$ starting at $x$. Let $A_{s}^{1}$ be the set of $x \in A_{s}$ that are leaves in $G_{s, s}$. For $x \in A_{s}^{1}$, let $p(x)$ be the lex-least path of length $n$ starting at $x$ in $G \backslash\left(G_{s, s} \cup H_{s-1}\right)$. Such a path exists since every vertex in $G$ has degree at least 2, and since if $y$ is a neighbor of $x$ that is not in $G_{s, s}$, then there is no simple path of length at most $d(0) \geqslant 3 n$ beginning $x, y, \ldots$ that ends in an element of $H_{s-1}$ by the definition of $G_{s, s}$. Let $J_{s}=\left\{p(x): x \in A_{s}^{0} \vee x \in A_{s}^{1}\right\}$ and let $G_{s}=G_{s, s} \cup J_{s}$. Clearly, $H_{s}$ satisfies (1) by definition.

Suppose $C$ is a connected component of $G_{s}$. We want to prove $C$ is $n$-spindly. Now $C$ contains a unique $x \in A_{s}$. We consider three cases. Case 1: If $x \in A_{s}^{0}$, then clearly $C$ is just a path of length $n$; hence, $C$ is $n$-spindly. Case 2: If $x \in A_{s}^{1}$, then let $p(x)=x, \ldots, z$ have endpoint $z$. In this case, $z$ is the distinguished leaf $C$; if $l$ is any other leaf of $C$, then $d(z, l) \geqslant n$ since $p(x)$ has length $n$. By the definition 
of $G_{s}$, any leaf in $C$ not equal to $z$ is the endpoint of a path from $G_{s, i-1}$ to $H_{s-1, s-i}$ for some $i$. Since any two connected components of $H_{s-1}$ have distance at least $2 n$ by (4), all these leaves have distance pairwise greater than $2 n$. So $C$ is $n$-spindly. Case 3: If $x \notin A_{s}^{0}$ and $x \notin A_{s}^{1}$, then all leaves of $C$ are endpoints of paths from $G_{s, i-1}$ to $H_{s-1}$ and have distance greater than $2 n$, so $C$ is $n$-spindly.

Now we verify parts (2) and (3) of the induction hypothesis. By the construction of $G_{s}$, every connected component of $G_{s}$ has diameter at most $2 d(s-1)+\cdots+$ $2 d(0)+n \leqslant d(s)-2 d(s-1)$. Since connected components of $H_{s-1}$ have diameter at most $d(s-1)$ by our induction hypothesis, connected components of $H_{s, s}$ therefore have diameter at most $d(s)$. Similarly, the distance between any two connected components of $G_{s}$ is at least $3 d(s)-2 d(s-1)-\cdots-2 d(0)-2 n \geqslant$ $2 d(s)+2 d(s-1)$. Hence, connected components of $H_{s, s}$ are pairwise of distance at least $2 d(s)$ since connected components of $H_{s-1}$ have diameter at most $d(s-1)$. Note that if $C$ is a connected component of $H_{s, k}$, then it is also a connected component of $H_{s^{\prime}, k}$ for all $s^{\prime}<s$. Hence, parts (2) and (3) of the induction hypothesis are also true for all $k<s$. This verifies parts (2) and (3) of the induction hypothesis.

Now we show that part (4) of the induction hypothesis holds. Suppose $C$ is a connected component of $H_{s, s}$. We want to show that distance from $y \in C$ to any other connected component $C^{\prime}$ of $H_{s, k}$ is greater than $2 n$ for $k \leqslant s$. When $k=s$, this follows from (3), so assume $k<s$. For a contradiction, let $y$ be a vertex in $C$ with $d\left(y, C^{\prime}\right) \leqslant 2 n$. We may assume that $y \in G_{s}$ since if $y \in G_{s^{\prime}}$ for $s^{\prime}<s$, then $d\left(y, C^{\prime}\right)$ follows from our induction hypothesis. We may further assume $y \in G_{s, s}$. To see this, let $x \in C$ be the unique vertex in $C$ with $x \in A_{s}$. If $x \in A_{s}^{0}$, then $C=p(x)$, and $d\left(x, H_{s-1}\right)>3 n$, so $d\left(y, H_{s-1}\right)>2 n$ since $p(x)$ has length $n$. If $x \in A_{s}^{1}$, then any path of length at most $2 n$ from $x \in p(z)$ to an element of $H_{s-1}$ must go through $y$ by our discussion after the definition of $p(x)$.

Hence, let $y \in G_{s, s}$ be so that $d\left(y, C^{\prime}\right) \leqslant 2 n$. Let $y^{\prime}$ be the closest element in $G_{s, s-k} \uparrow C$ to $C^{\prime}$. Hence, by the construction of $G_{s, s}$, we have $d\left(y, y^{\prime}\right) \leqslant$ $d(k-1)+\cdots+d(0)$. Since $C^{\prime}$ is of distance at most $2 n$ from $y, C^{\prime}$ is of distance at most $2 n+d(k-1)+\cdots+d(0)<d(k)$ from $x^{\prime}$. First, suppose $x^{\prime}$ is also a vertex in $G_{s, s-k-1}$. Then $x^{\prime}$ would be an element of $G_{s, s-k-1}$ of distance $<d(k)$ from an element of $H_{s, k}$, and so in the definition of $G_{s, s-k}$, there should have been a path added from $G_{s, s-k-1}$ to $C^{\prime}$ in $G_{s, s-k}$. If $x^{\prime}$ is not a vertex in $G_{s, s-k-1}$, then $x^{\prime}$ must be part of a path added in $G_{s, s-k}$ from an element of $G_{s, s-k-1}$ to some connected component $C^{\prime \prime}$ of $H_{s-1, k}$. Since this path is of distance at most $d(k)$, this would imply that $C^{\prime}$ and $C^{\prime \prime}$ are of distance $<2 d(k)$, which contradicts part (3) of the induction hypothesis.

We are now ready to prove our path decomposition lemma. 
Lemma 3.4. Suppose $G$ is a locally finite acyclic graph on a Polish space and $n \geqslant 1$. Then there is a comeager Borel set $D$ such that $G \uparrow D$ has a path decomposition of length at least $n$.

Proof. We prove this lemma by combining Lemmas 3.3 and 3.1 with the obvious derivative process to obtain sets of paths.

Suppose $\left(D_{i}\right)_{i \in \mathbb{N}}$ is such that each $S \in D_{i}$ is a finite sequence of paths in $G$ that are end-ordered, and $\left(D_{i}\right)_{i \in \mathbb{N}}$ is end-ordered. Let $<_{\left(D_{i}\right)}$ (suppressing the indexing for clarity) be the partial order on the paths appearing in the elements of $D_{i}$ where $p<_{{ }_{\left(D_{i}\right)}} p^{\prime}$ if $p, p^{\prime}$ share some vertex, and either $p, p^{\prime}$ both appear in some sequence $S \in D_{i}$ where $p$ appears before $p^{\prime}$ or $p$ is in an element of $D_{i}$ and $p^{\prime}$ is in an element of $D_{j}$ for $i<j$.

We begin by applying Lemma 3.3 to obtain an $n$-spindly decomposition $G_{0}$, $G_{1}, \ldots$ of $G$ restricted to some comeager $G$-invariant Borel set. If $C$ is an $n$ spindly connected component of some $G_{i}$, let $P(C)$ be the lexicographically least decomposition $\left(p_{0}, \ldots, p_{k}\right)$ of $C$ satisfying the conclusion of Lemma 3.1. Letting $D_{i, 0}=\left\{P(C)\right.$ : $C$ is a connected component of $\left.G_{i}\right\}$, we obtain a sequence $\left(D_{i, 0}\right)_{i \in \mathbb{N}}$ of sets of finite sequences of paths in $G$, and the associated partial order $<_{\left(D_{i, 0}\right)}$ defined in the previous paragraph.

Inductively, for $j \geqslant 0$, let $P_{j}$ be the set of $p$ appearing in some element of $D_{i, j}$ such that there is no $p^{\prime}<_{\left(D_{i, j}\right)} p$. Then let $D_{i, j+1}$ be the set of all sequences in $D_{i, j}$ with all elements of $P_{j}$ removed. These $P_{j}$ are our desired set of paths. Every path $p^{\prime}$ in each $S \in D_{i}$ must eventually appear in some $P_{j}$ since there are only finitely many $p$ such that $p<_{\left(D_{i, 0}\right)} p^{\prime}$.

A useful observation is that if $G$ is a graph with a path decomposition, the decomposition may be assumed to consist of paths of bounded length. This follows the fact that the intersection graph on paths has a countable Borel coloring, and a derivative operation analogous to that of Lemma 3.4.

LEMma 3.5. Suppose $G$ is a locally finite Borel graph with a Borel path decomposition $P_{0}, P_{1}, \ldots$ of length at least $n$. Then $G$ admits a Borel path decomposition $P_{0}^{\prime}, P_{1}^{\prime}, \ldots$ of length at least $n$ such that every path $p \in P_{i}^{\prime}$ has length at most $2 n$.

Proof. Every path of length greater than $2 n$ can clearly be written as a finite union of paths of length between $n$ and $2 n$. Hence, we may replace any path $p \in P_{i}$ of length greater than $2 n$ by the lex-least finite set of paths of length between $n$ and $2 n$ whose union is $p$. This gives a sequence $P_{0}, P_{1}, \ldots$ having every property of being a Borel path decomposition with the exception that $P_{i}$ may not consist of 
vertex-disjoint paths (but with the property that every path in every $P_{i}$ has length at most $2 n$ ).

Let $H$ be the graph on the paths $\bigcup_{i} P_{i}$, where distinct $p, p^{\prime} \in \bigcup_{i} P_{i}$ are adjacent in $H$ if they share some vertex. Then $H$ is a locally finite Borel graph and hence has a countable Borel coloring $c: \bigcup_{i} P_{i} \rightarrow \mathbb{N}$ by [KST, Proposition 4.10].

Inductively, let $D_{i, 0}=P_{i}$. For a fixed $j$, we can order the paths in $\bigcup_{i} D_{i, j}$ by $p<_{\left(D_{i, j}\right)} p^{\prime}$ if $p \in D_{i, j}$ and $p^{\prime} \in D_{i^{\prime}, j}$, where either $i<i^{\prime}$ or $i=i^{\prime}$ and $c(p)<c\left(p^{\prime}\right)$. Now a construction identical to the last paragraph of the proof of Lemma 3.4 gives our desired Borel path decomposition.

LEMMA 3.6. Suppose that $E$ is an abstract system of congruences on $n$, which is nonexpanding, and $R=\left\{\left(S_{1}, T_{1}\right), \ldots,\left(S_{k}, T_{k}\right)\right\}$ is a minimal good generating set of E. Suppose also that a is a free Borel action of the group

$$
\Gamma=\left\langle\gamma_{1} \cdots \gamma_{k} \mid\left\{\gamma_{i}^{2}=1: T_{i}=S_{i}^{c}\right\}\right\rangle
$$

on a Polish space X. If $G\left(a,\left\{\gamma_{1}, \ldots, \gamma_{k}\right\}\right)$ has a Borel path decomposition of length at least $r$ for sufficiently large $r$ (depending on $E$ ), then there is an arealization of $E$ with Borel pieces witnessed by

$$
\gamma_{i} \cdot \bigcup_{j \in S_{i}} A_{j}=\bigcup_{j \in T_{i}} A_{j} .
$$

Furthermore, if the space $X$ is assumed to be perfect, then the sets $A_{1}, \ldots, A_{k}$ can be chosen so that each is nonmeager.

Proof. Let $G$ be the graph $G=G\left(a,\left\{\gamma_{1}, \ldots, \gamma_{k}\right\}\right)$. The idea of our proof is as follows. We first argue that there is a sufficiently large length $r$ so that given any path $p$ of length at least $r$ in $G$, if we have already assigned the endpoints of $p$ to be in elements of $A_{0}, \ldots, A_{n-1}$, then there is some way of consistently assigning the interior points of the path to elements of $A_{0}, \ldots, A_{n-1}$ so as to obey the congruences required in $(*)$. Then we use a path decomposition of length at least $r$ for $G$ to inductively construct a realization of this system of congruences.

Suppose that $g=g_{l} \ldots g_{0}$ is a reduced word in $\Gamma$, where $g_{i} \in\left\{\gamma_{1}^{ \pm}, \ldots, \gamma_{k}^{ \pm}\right\}$ are generators. If we begin at some $x \in X$, then such a reduced word of length $l+1$ gives a path of length $l+1$ in $G$ : the path $x, g_{0} \cdot x, \ldots, g_{l} \cdots g_{0} \cdot x$. We give a definition concerning what elements of $A_{0}, \ldots, A_{n-1}$ the elements of this path can belong to. Define functions $X$ and $Y$ on generators as follows: $X\left(\gamma_{j}\right)=$ $S_{j}, Y\left(\gamma_{j}\right)=T_{j}, X\left(\gamma_{j}^{-1}\right)=T_{j}$, and $Y\left(\gamma_{j}^{-1}\right)=S_{j}$. We say that $n_{0}, \ldots, n_{l+1}$ is a labeling of $g=g_{l} \cdots g_{0}$ if for all $i$, we have $n_{i} \in X\left(g_{i}\right)$ if and only if $n_{i+1} \in Y\left(g_{i}\right)$. So labelings correspond to acceptable assignments of the points $x, g_{0} \cdot x, \ldots$, $g_{l} \cdots g_{0} \cdot x$ to the sets $A_{0}, \ldots, A_{n-1}$. 
We are interested in the ways labelings of $g$ may start and end. If $k, m \in n$, we say a reduced word $g$ is $(k, m)$-bad if there is no labeling $n_{0}, \ldots, n_{l+1}$ of $g$ with $n_{0}=m$ and $n_{l+1}=k$. We say that $g$ is bad if there is some $k, m \in n$ such that $g$ is $(k, m)$-bad. We will use a pigeonhole principle argument to show there is a bound on the length of bad words.

To begin, note that if $g=g_{l} \cdots g_{0}$ is bad, then $g_{l} \cdots g_{1}$ and $g_{l-1} \cdots g_{0}$ are also bad. That is, initial segments and final segments of bad words are bad.

Suppose $g=g_{l} \cdots g_{0}$ is $(k, m)$-bad. Then exactly one of the following holds. Either

(1) $m \in Y\left(g_{l}\right)$ and $g$ is $\left(k, m^{\prime}\right)$-bad for every $m^{\prime} \in Y\left(g_{l}\right)$ or

(2) $m \in Y\left(g_{l}\right)^{\mathrm{c}}$ and $g$ is $\left(k, m^{\prime}\right)$-bad for every $m^{\prime} \in Y\left(g_{l}\right)^{\mathrm{c}}$.

Fix a $(k, m)$-bad word $g$. Define a pair of associated sequences $V_{g, k}(i)$ and $W_{g, k}(i)$, where $\left(V_{g, k}(i), W_{g, k}(i)\right)=\left(X\left(g_{i}\right)^{\mathrm{c}}, Y\left(g_{i}\right)^{\mathrm{c}}\right)$ if $g_{i} \cdots g_{0}$ is $\left(k, m^{\prime}\right)$-bad for every $m^{\prime} \in Y\left(g_{i}\right)^{\mathrm{c}}$ and $\left(V_{g, k}(i), W_{g, k}(i)\right)=\left(X\left(g_{i}\right), Y\left(g_{i}\right)\right)$ otherwise. It is clear that there exist labelings $n_{0}, \ldots, n_{l+1}$ of $g$, where $n_{i} \in V_{g, k}(i)$ and $n_{i+1} \in W_{g, k}(i)$ for every $i$. Indeed, we have that $V_{g, k}(i) E W_{g, k}(i)$ by definition, and $W_{g, k}(i) \subseteq$ $V_{g, k}(i+1)$ for all $i \leqslant l$ or else $g$ is not a bad word.

Suppose for a contradiction that there are infinitely many bad words. We divide this into two cases.

Case 1: Suppose that there are arbitrarily long bad words $g$ such that $g$ is $(k, m)$ bad for some $(k, m)$, and $W_{g, k}(i)=V_{g, k}(i+1)$ for all $i<l$. Hence, $V_{g, k}(0) E$ $V_{g, k}(1) E \cdots E V_{g, k}(l)$. By the pigeonhole principle, and since initial segments and final segments of bad words are bad, we can find some bad word $g$ such that $g$ is $(k, m)$-bad, and

$g$ has length at least 2 and $V_{g, k}(0)=W_{g, k}(l)$ or $V_{g, k}(0)=W_{g, k}(l)^{\mathrm{c}}$.

We claim that this implies that either the word $g$ is not reduced or the generating set of $E$ is not a minimal good generating set.

First, we may assume that $g$ has minimal length among bad words with property $(* *)$, and so no proper subword of $g$ has property $(* *)$.

If $V_{g, k}(0)=W_{g, k}(l)$, then the minimal length of $g$ among words with (**) implies that $g_{0} \neq g_{i}^{ \pm 1}$ for any $i>0$. This implies that the generating set $R$ is not a minimal good generating set; the fact that $V_{g, k}(0) E V_{g, m}(1)$ follows from $V_{g, k}(1) E W_{g, k}(1)=V_{g, k}(2) E \cdots E V_{g, k}(l) E W_{g, k}(l)$ and $W_{g, k}(l)=V_{g, k}(0)$. In particular, removing the pair $\left(S_{j}, T_{j}\right)$, where $g_{0}=\gamma_{j}$, would still generate $E$. Hence, the generating set is not minimal.

In the case where $V_{g, k}(0)=W_{g, k}(l)^{\mathrm{c}}$, we can also remove the pair $\left(S_{j}\right.$, $\left.T_{j}\right)$, where $g_{0}=\gamma_{j}$, since there must be a generator witnessing $V_{g, k}(0) E$ 
$V_{g, k}(0)^{\mathrm{c}}=W_{g, k}(l)$ by our definition of a good generating set (see Definition 2.2). In particular, a good generating set must contain every relation of the form $\left(S, S^{\mathrm{c}}\right)$ where $S E S^{\mathrm{c}}$.

Case 2: Suppose case 1 does not hold. Then by the pigeonhole principle, and since initial segments and final segments of bad words are bad, we can find some $(k, m)$-bad word $g$ such that $V_{g, k}(0)=W_{g, k}(l+1)$, and $W_{g, k}(i) \subsetneq V_{g, k}(i+1)$ for some $i \leqslant l$. Then we can obtain a contradiction to the nonexpansion of $E$ by cyclically permuting the sequences to bring $V_{g, k}(i+1)$ to the 0 th position and $W_{g, k}(i)$ to the $l$ th position.

This finishes the proof that there are only finitely many bad words.

Now let $r$ be sufficiently large so that there are no bad words of length $r$, and let $P_{0}, P_{1}, \ldots$ be a Borel path decomposition of $G$ of length at least $r$. We may assume that this path decomposition satisfies the conclusion of Lemma 3.5. Now we inductively construct a Borel $a$-realization $A_{0}, \ldots, A_{n-1}$ of $E$ in countably many steps. After step $i$, we will have assigned each vertex appearing in the paths in $P_{j}$ for $j \leqslant i$ to some $A_{0}, \ldots, A_{n-1}$.

At step $i$, we will consider the paths $p \in P_{i}$. For each such path $p$, we assign the vertices of $p$ to be the lex-least assignment to $A_{0}, \ldots, A_{n-1}$ that is consistent with the requirement (*) in the statement of the lemma. There is guaranteed to be such an assignment since we will have assigned at most the start and end nodes of the path to $A_{0}, \ldots, A_{n-1}$ and since the path has length at least $r$, the group element corresponding to it is not bad.

At the end of this construction, we will have assigned every element of $X$ to some $A_{0}, \ldots, A_{n-1}$. Since every edge in $G$ appears in some path $p$, this ensures that the requirement $(*)$ is satisfied at the end of the construction.

To finish, we prove the 'furthermore' statement at the end of the lemma. Suppose that the space $X$ is perfect. We show that the sets $A_{1}, \ldots, A_{n}$ can be chosen to be nonmeager. Note that it suffices to have a path decomposition, where the first set $P_{0}$ of paths has a set of endpoints $D$ that is nonmeager. If this is the case, then we may partition $D$ into $k$ many nonmeager Borel sets since $X$ is perfect. Then we may assign these $k$ sets to $A_{1}, \ldots, A_{k}$. This is because in our construction above, the endpoints of the paths of $P_{0}$ may be assigned to $A_{1}, \ldots, A_{k}$ arbitrarily.

So we need to show that we can construct a path decomposition, where the set of endpoints of paths in $P_{0}$ is nonmeager. To see this, observe that in our proof of Lemma 3.3 given the subsets $\left(A_{i}\right)_{i \in \mathbb{N}}$ of $X$ such that the elements of $A_{i}$ are pairwise of distance greater than $d(i)$, all the elements of the set $A_{0}$ become endpoints of paths in $P_{0}$ in the final path decomposition. Hence, it suffices to show that $A_{0}$ can be chosen to be nonmeager in [MU16, Lemma 3.1]. To see this, note first that we can find a Borel nonmeager $k$-independent set. This is because $G^{\leqslant k}$ 
has a countable Borel coloring [KST, Proposition 4.10] and one of the color sets must therefore be a nonmeager $k$-independent Borel set $A_{0}$. Now apply [MU16, Lemma 3.1] to the graph $G \backslash A_{0}$ and the function $f(n)=d(n+1)$.

We are now ready to prove Theorem 1.2.

Proof of Theorem 1.2. We begin with the forward direction of Theorem 1.2. Suppose $A_{0}, \ldots, A_{n-1}$ is a Baire measurable realization of an abstract system of congruences $E$ on $n$, where every $A_{i}$ is nonmeager. By Theorem 1.1, it suffices to show that $E$ is nonexpanding. For a contradiction, suppose there are sequences of sets $\left(V_{i}\right)_{i \leqslant k}$ and $\left(W_{i}\right)_{i \leqslant k}$ with $V_{i}, W_{i} \in \mathcal{P}_{\text {pr }}(m)$ such that $V_{i} E W_{i}$ for every $i \leqslant k$, $W_{i} \subseteq V_{i+1}$ for every $i<k$ and $V_{0} \supsetneq W_{k}$. Let $A=\bigcup_{i \in V_{0}} A_{i}$ and $B=\bigcup_{i \in W_{k}} A_{i}$. Let $\gamma$ be the product of the group elements witnessing $V_{i} E W_{i}$ taken in increasing order for $i \leqslant k$. It follows that $\gamma \cdot A \subseteq B$. Clearly, if $x \in A \backslash B$, then for all $n>0$, $\gamma^{n} \cdot x \notin A \backslash B$.

Now there are two cases. First, if the rotation given by $\gamma$ is rational (that is, periodic), this implies that $A \backslash B$ is not in any orbit of $\gamma$. This contradicts the fact that $A \backslash B$ is nonmeager.

Second, suppose the rotation of $\gamma$ is aperiodic. Then $A \backslash B$ meets each orbit of $\gamma$ in at most one point, which contradicts $A \backslash B$ being nonmeager as follows. If $A \backslash B$ was nonmeager, there would be an open set $U$ in which $A \backslash B$ is comeager. But since $\gamma$ is an irrational rotation, we can find some $n>0$ rendering $\gamma^{n}$ arbitrarily close to the identity, and hence some $n$ for which $\gamma^{n} U \cap U \neq \varnothing$. Since $\gamma$ is a homeomorphism, this implies that both $A \backslash B$ and $\gamma^{n} \cdot(A \backslash B)$ are comeager in $\gamma^{n} U \cap U$. But then there is some $x$ so that $x \in A \backslash B$ and $\gamma^{n} \cdot x \in A \backslash B$, which is a contradiction. This finishes the proof of the forward implications.

To prove the reverse implication, suppose that $E$ is noncomplementing and nonexpanding. Choose some $R=\left\{\left(S_{1} T_{1}\right), \ldots,\left(S_{k} T_{k}\right)\right\}$ that minimally generates $E$, and let $\left\langle\gamma_{1} \cdots \gamma_{k}\right\rangle$ be rotations of the 2 -sphere that generate a copy of $\mathbb{F}_{k}$.

Now let $r$ be sufficiently large (so as to satisfy the hypothesis of Lemma 3.6). By Lemma 3.4, we can find a comeager $G$-invariant Borel set $D$ so that there is a Borel path decomposition of length at least $r$ of $G \uparrow D$. Let $a^{\prime}$ be the restriction of the action of $\left\langle\gamma_{1}, \ldots, \gamma_{k}\right\rangle$ to $D$. Then by Lemma 3.4, we can find a Borel $a^{\prime}$ realization $A_{0}^{\prime}, \ldots, A_{n-1}^{\prime}$ of $E$. By the 'furthermore' clause of Lemma 3.6, we can assume each of $A_{0}^{\prime}, \ldots, A_{n-1}^{\prime}$ to be nonmeager.

By Lemma 2.3, there is some realization $A_{0}^{\prime \prime}, \ldots, A_{n-1}^{\prime \prime}$ of $E$ on the 2-sphere witnessed using (*). To finish our proof, replace $A_{i}^{\prime \prime}$ with $A_{i}^{\prime}$ on $D$ to obtain a Baire measurable realization of $E$ on the 2-sphere. That is, set $A_{i}=\left(A_{i}^{\prime \prime} \cap D^{\mathrm{c}}\right) \cup$ $\left(A_{i}^{\prime} \cap D\right)$. 


\section{Borel path decompositions from Borel end selections}

Suppose $f: X \rightarrow X$. We say that $f$ is aperiodic if for all $x \in X$ and $n \geqslant 1$, we have $f^{n}(x) \neq x$. Let $G_{f}$ be the graph induced by $f$, where distinct $x_{0}, x_{1} \in X$ are $G_{f}$-adjacent if $f\left(x_{0}\right)=x_{1}$ or $f\left(x_{1}\right)=x_{0}$. Suppose $A \subseteq X$. We say that $A$ is forward recurrent (with respect to $f$ ) if for every $x \in X$, there exists some $n \geqslant 0$ such that $f^{n}(x) \in A$.

We have the following lemma showing that bounded-to-one Borel functions admit forward recurrent $r$-independent sets. Recall that a function $f: X \rightarrow Y$ is bounded-to-one if there is some $k>0$ such that for every $y \in Y,\left|f^{-1}(y)\right| \leqslant k$.

Lemma 4.1. Suppose $X$ is a standard Borel space and $f: X \rightarrow X$ is an aperiodic bounded-to-one Borel function. Then for every $r \geqslant 1$ there exists a Borel set $A \subseteq X$ that is forward recurrent and $r$-independent.

Proof. Let $G_{f}^{\leqslant r}$ be the graph on $X$, where distinct $x, y \in X$ are $G_{f}^{\leqslant r}$-adjacent if $d(x, y) \leqslant r$. Since $G_{f}$ has bounded degree, $G_{f}^{\leqslant r}$ also has bounded degree. Hence, by [KST, Theorem 4.6], there is a Borel coloring $c$ of $G_{f}^{\leqslant r}$ with finitely many colors. Let $A$ be the set of $x \in X$ such that $c(x)$ is equal to the least number appearing infinitely often in the sequence $c(x), c(f(x)), c\left(f^{2}(x)\right) \cdots$. Then for each $x$, all the elements of $A$ in the $(G$-)connected component of $x$ have the same color, and hence $A$ is $r$-independent since $c$ is a coloring of $G_{f}^{\leqslant r} . A$ is forward recurrent by construction.

Now we show that we can obtain Borel path decompositions from Borel end selections.

LEMMA 4.2. Suppose $G$ is an acyclic bounded degree Borel graph on X such that there is a Borel selection of finitely many ends in every connected component of $G$. Then for every $n>0, G$ admits a Borel path decomposition of length at least $n$.

Proof. We are given a bounded degree acyclic Borel graph $G$ on a standard Borel space $X$, where every vertex has degree at least 2 . First, by [HM, Theorem C], which builds on methods from [Mi], if there is a Borel function selecting finitely many ends from every connected component of $G$, then there is a Borel function selecting one or two ends in every connected component of $G$. Hence, we can partition $X$ into two $G$-invariant Borel sets $C_{1}, C_{2}$ so that $G \uparrow C_{1}$ has a Borel selection of one end in each connected component and $G \uparrow C_{2}$ has a Borel selection of two ends in each connected component. 
Let $r(x)$ be the Borel function selecting one end in each connected component of $G\left\lceil C_{1}\right.$. We may assume that $r(x)$ begins with the vertex $x$ (by either appending the path from $x$ to the start of the ray $r(x)$ if $x$ is not included in the ray or deleting the vertices preceding $x$ if $x$ is included in the ray). Let $f(x)$ be the vertex after $x$ in $r(x)$. Then it is easy to see that $f: C_{1} \rightarrow C_{1}$ generates the graph $G$.

Let $B_{2} \subseteq C_{2}$ be the Borel set of vertices lying on the geodesic between the two ends chosen in $C_{2}$. Precisely, let $r_{0}(x), r_{1}(x)$ be the functions selecting two ends in each connected component of $G \uparrow C_{2}$. We may similarly assume that $r_{0}(x)$ and $r_{1}(x)$ begin with the vertex $x$, and let $f_{0}(x)$ be the vertex after $x$ in $r_{0}(x)$ and $f_{1}(x)$ be the vertex after $x$ in $r_{1}(x)$. Then $B_{2}=\left\{x \in C_{2}: f_{0}(x) \neq f_{1}(x)\right\}$. It is easy to see that every connected component of $G \uparrow B_{2}$ is 2-regular and every connected component of $G\left\lceil C_{2}\right.$ contains exactly one connected component of $G\left\lceil B_{2}\right.$.

By Lemma 4.1, we can find a forward recurrent Borel set $A \subseteq C_{1}$ such that $A$ is $2 n$-independent in $G$. Let $P_{0}^{1}$ be the set of lex-least paths of length $n$, which begin at some vertex of $A$, and let $B_{1}$ be the set of vertices contained in some element of $P_{0}^{1}$. If $x \in C_{1} \backslash B_{1}$, let $[x]$ be the set of vertices $y$ for which there is a path $p$ from $x$ to $y$ for which no interior vertex of $p$ is in $B_{1}$. The forward recurrence of $A$ implies that for every $x \in C_{1}$, there is a unique forwardmost element of $[x]$ under $f$. It is also clear that $G\lceil[x]$ satisfies the hypothesis of Remark 3.2. For each $x$, the space of $n$-spindly decompositions is a compact space in the natural topology on all such decompositions. Hence, by compact uniformization ([Sr, Theorem 5.7.1], see also [K, Theorem 18.18]), there is a Borel way of selecting a unique path decomposition of length at least $n$ for $G\left\lceil[x]\right.$ for each $x \in C_{1} \backslash B_{1}$. Hence, we can extend $P_{0}^{1}$ to a Borel path decomposition of length at least $n$ for $G \uparrow C_{1}$.

On $G \uparrow C_{2}$, we can first partition $G \uparrow B_{2}$ into a Borel set $P_{0}^{2}$ of finite paths of length at least $n$. If $x \in C_{2} \backslash B_{2}$, let $[x]$ be the set of $y \in X$ such that there is a path $p$ from $x$ to $y$ for which no interior vertex of $p$ is in $B_{2}$. Once again, $G\lceil[x]$ is $n$ spindly. Hence, by Remark 3.2, we can extend $P_{0}^{2}$ to a Borel path decomposition of length at least $n$ for $G \uparrow C_{2}$.

Using the Adams end selection, we can use this lemma to show that $\mu$ hyperfinite free actions of $\mathbb{F}_{n}$ have $\mu$-measurable realizations of abstract systems of congruences that are noncomplementing and nonexpanding.

THEOREM 4.3. Suppose that $n \geqslant 2$, and a is a free Borel action of $\mathbb{F}_{n}$ on a standard probability space $(X, \mu)$ that is $\mu$-hyperfinite. Then there is a $\mu$ measurable a-realization of every abstract system of congruences $E$ that is noncomplementing and nonexpanding.

Proof. Let $R$ minimally generate $E$. Pass to a free subgroup $\mathbb{F}_{k} \leqslant \mathbb{F}_{n}$, where $k=|R|$. Let $S$ be the set of generators of $S$. By a theorem of Adams [JKL, 
Lemma 3.21], on a conull set there is a Borel function selecting either one or two ends from each connected component of $G(a, S)$. Hence, the theorem follows from Lemmas 4.2 and 3.6.

When we apply Lemma 4.2, it will be useful to know that end selections pass between finite index subgroups.

LEMma 4.4. Suppose a is a free Borel action of a finitely generated group $\Gamma$ on $X$. Let $\Delta \leqslant \Gamma$ be a finitely generated finite index subgroup of $\Gamma$ and $b$ be the restriction of the action of a to $\Delta$. Then if $S \subseteq \Gamma$ and $R \subseteq \Delta$ are finite symmetric generating sets, then $G(a, S)$ has a Borel selection of finitely many ends if and only if $G(b, R)$ has a Borel selection of finitely many ends.

Proof. Since $\Delta$ is a finite index in $\Gamma$, each $G(a, S)$ connected component contains finitely many components of $G(b, R)$ and each connected component of $G(b, R)$ is bounded distance from every point in the connected component of $G(a, S)$ it is contained in. Hence, there is an effectively defined bijection between ends in a connected component of $G(a, S)$ and ends in each $G(b, R)$-component that it contains.

More precisely, suppose $r=\left(x_{i}\right)_{i \in \mathbb{N}}$ is a ray representing an end in $G(a, S)$ and $C$ is a connected component of $G(b, R)$. We define a ray $f_{C}(r)$ in $G(b, R)\lceil C$ as follows. To each $x_{i}$, we associate the nearest point $y_{i}$ in $C$, and let $f_{C}(r)$ be the lex-least ray passing through all the points $\left(y_{i}\right)_{i \in \mathbb{N}}$, erasing loops. The map $f_{C}$ clearly lifts to a map sending a selection of finitely many ends in $G(a, S)$ to a selection of finitely many ends in $G(b, R)$. The reverse implication is similar.

\section{Constructive realizations of nonexpanding abstract systems of congruences for $\mathrm{PSL}_{2}(\mathbb{Z})$ acting on $\mathrm{P}^{1}(R)$}

The group $\mathrm{PSL}_{2}(\mathbb{Z})$ acts on the space $\mathrm{P}^{1}(\mathbb{R})$ of lines in $\mathbb{R}^{2}$ through the origin. By identifying such a line with the $x$-value $x \in \mathbb{R} \cup\{\infty\}$ of its intersection point with the line $y=1$, it is easy to see that this action is isomorphic to the action of $\mathrm{PSL}_{2}(\mathbb{Z})$ on $\mathbb{R} \cup\{\infty\}$ by fractional linear transformations, where $\left[\begin{array}{ll}a & b \\ c & d\end{array}\right]$ acts via $x \mapsto \frac{a x+b}{c x+d}$.

It is a standard fact (see [Se, VII.1]) that $\mathrm{PSL}_{2}(\mathbb{Z})$ is generated by the two transformations $\alpha(x)=x+1$ and $\beta(x)=-1 / x$ and, moreover, that it factors as the free product of $\langle\beta\rangle$ of order 2 and $\langle\alpha \beta\rangle$ of order 3 .

The group $\mathrm{PGL}_{2}(\mathbb{Z})$ has index 2 over $\mathrm{PSL}_{2}(\mathbb{Z})$ and, similarly, is generated by $\alpha(x)=x+1$ and $\gamma(x)=1 / x$. Note that $\beta(x)=\alpha^{-1}\left(\gamma\left(\alpha\left(\beta\left(\alpha^{-1}(x)\right)\right)\right)\right)=$ $(-1+1 /(1+1 /(x-1)))=-1 / x$. 
Let Irr denote the set of irrational numbers. Each $x \in \operatorname{Irr}$ has a unique continued fraction expansion

$$
x=a_{0}+\frac{1}{a_{1}+\frac{1}{a_{2}+\frac{1}{a_{3}+\cdots}}},
$$

where $a_{0} \in \mathbb{Z}$ and $a_{1}, a_{2}, \ldots \in \mathbb{Z}^{+}$are positive integers. We note the continued fraction expansion of $x$ as $\left(a_{0} ; a_{1}, \ldots\right)$. The following lemma is standard.

LEMMA 5.1. Let $f:$ Irr $\rightarrow$ Irr be the function given by

$$
f(x)= \begin{cases}x-1 & \text { if } x>0 \\ 1 / x & \text { if } x \in(0,1), \\ x+1 & \text { if } x<0\end{cases}
$$

Then $f$ generates the orbit equivalence relation of $\mathrm{PGL}_{2}(\mathbb{Z})$ on $\operatorname{Irr}$, and so $x$, $y \in \operatorname{Irr}$ are in the same orbit if and only if their continued fraction expansions are tail-equivalent.

Proof. The equivalence relation generated by $f$ is clearly contained in the orbit equivalence relation of $\mathrm{PGL}_{2}(\mathbb{Z})$ since $f$ is defined piecewise by fractional linear transformations.

Recall that two continued fraction expansions $\left(a_{0} ; a_{1}, \ldots\right)$ and $\left(b_{0} ; b_{1}, \ldots\right)$ are tail-equivalent if there exists some $n, m>0$ such that $a_{n+i}=b_{m+i}$ for all $i \geqslant 0$. Since

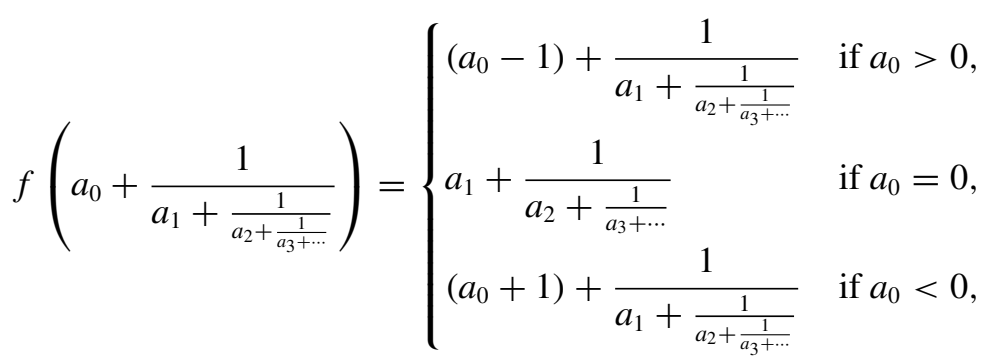

it is clear that if $x$ and $y$ are tail-equivalent, then they are in the same equivalence class of the equivalence relation generated by $f$.

To finish, since $\alpha(x)=x+1$ and $\gamma(x)=1 / x$ generate $\mathrm{PGL}_{2}(\mathbb{Z})$, it suffices to show that if $x \in \operatorname{Irr}$, then $x, x+1$, and $1 / x$ are tail-equivalent. It is trivial to see that $x$ and $x+1$ are tail-equivalent. That $x$ and $1 / x$ are tail-equivalent is clear when $x>0$. When $x<0$, since either $x$ or $1 / x$ is less than -1 , by swapping $x$ and $1 / x$, we may assume the continued fraction expansion of $x$ is $x=a+\frac{1}{b+C}$, 
where $a \leqslant-2$ and $b \geqslant 1$. Then, we apply the following identity:

$$
\frac{1}{a+\frac{1}{b+C}}=-1+\frac{1}{1+\frac{1}{(-a-2)+\frac{1}{1+\frac{1}{(b-1)+C}}}} .
$$

Note that $-a-2 \geqslant 0$ and $b-1 \geqslant 0$. If either of these two terms is equal to zero, this just removes the corresponding term in the continued fraction expansion since $\frac{1}{0+\frac{1}{a_{n}+C}}=a_{n}+C$.

COROLlaRY 5.2. Let a be the restriction of the action of $\mathrm{PSL}_{2}(\mathbb{Z})$ to the irrationals. Let $S=\{\alpha, \beta\}$ be the set of generators $\alpha(x)=x+1$ and $\beta(x)=-1 / x$. Then there is a Borel selection of one end in each equivalence class of $G(a, S)$.

Proof. By Lemma 5.1, there is a Borel selection of one end in the graph $G\left(a^{\prime}\right.$, $\{\alpha, \gamma\})$, where $a^{\prime}$ is the action of $\mathrm{PGL}_{2}(\mathbb{Z})$ on Irr, and $\gamma(x)=1 / x$. Hence, this corollary follows from Lemma 4.4 since $\mathrm{PGL}_{2}(\mathbb{Z})$ is index 2 over $\mathrm{PSL}_{2}(\mathbb{Z})$.

The action of $\mathrm{PSL}_{2}(\mathbb{Z})$ is free modulo a countable set since if $x=(a x+$ $b) /(c x+d)$, then $x$ is the solution to a quadratic equation with integer coefficients. To finish, we need to analyze the countable set on which the action is nonfree.

LEMma 5.3. For every $x \in P^{1}(\mathbb{R})$, the stabilizer $\operatorname{Stab}(x)$ of $x$ in $\mathrm{PSL}_{2}(\mathbb{Z})$ is cyclic.

Proof. It suffices to show for all $x$ that $\operatorname{Stab}(x)$ is a solvable subgroup of $\mathrm{PSL}_{2}(\mathbb{Z})$ containing no involution. Indeed, as $\mathrm{PSL}_{2}(\mathbb{Z}) \cong(\mathbb{Z} / 2 \mathbb{Z}) *(\mathbb{Z} / 3 \mathbb{Z})$, it follows from the Kurosh subgroup theorem [C, Theorem 7.8] that all solvable subgroups are either cyclic or the free product of two involutions, and we are done upon precluding the latter alternative.

Toward that end, first observe that the action of $\mathrm{PGL}_{2}(\mathbb{R})$ on $P^{1}(\mathbb{R})$ is transitive, and thus all stabilizers are conjugate to the stabilizer of the point at infinity. This stabilizer is isomorphic to the group of affine transformations of the real line and, in particular, is solvable. Returning to $\mathrm{PSL}_{2}(\mathbb{Z})$, it follows that the stabilizer of every point is a subgroup of a solvable group, and hence is itself solvable.

It remains to show that every nontrivial involution in $P G L_{2}(\mathbb{Z})$ acts freely on $P^{1}(\mathbb{R})$. But this is immediate as all such involutions are conjugate to $\beta: x \mapsto-1 / x$, which has no fixed point.

We can now prove Theorem 1.5 from Section 1. 
Proof of Theorem 1.5. Let $R$ be a minimal relation generating $E$. Let $k=|R|$. There is a finite index copy of $\mathbb{F}_{2}$ in $\mathrm{PSL}_{2}(\mathbb{Z})$ and hence a finite index copy of $\mathbb{F}_{k}$. Let the free generating set of $\mathbb{F}_{k}$ be $S$. Let $a$ be the restriction of the action of $\mathrm{PSL}_{2}(\mathbb{Z})$ to this copy of $\mathbb{F}_{k}$. Let $F \subseteq X$ be the subset on which the action of $F$ is free. By Lemma 5.3, the action of $\mathrm{PSL}_{2}(\mathbb{Z})$ on $F$ has cyclic stabilizers, and so by Lemma 2.3, there is a realization of $E$ witnessed by letting the generators $S$ of $F_{k}$ witness the elements of $R$. Since $X \backslash F$ is a subset of the quadratic rationals, it is countable, and so the sets realizing $E$ on $X \backslash F$ are Borel.

Now on $F$, the graph $G(a\lceil F, S)$ has a Borel selection of finitely many ends by Corollary 5.2 and Lemma 4.4. Hence, by Lemma 4.2, we have a Borel path decomposition and hence by Lemma 3.6, there is a realization of $E$ on $a \uparrow F$ once again with the $i$ th generator witnessing the $i$ th congruence in $R$. The theorem follows by taking the union of these two realizations.

\section{Applications of path decompositions in Borel combinatorics}

If $G$ is a locally finite acyclic Borel graph, then path decompositions for $G$ give a very strong type of unfriendly coloring.

LEMMA 6.1. Suppose $G$ is a locally finite acyclic Borel graph on $X$ where every vertex has degree at least 2. Then if $G$ has a Borel path decomposition of length at least 4, then $G$ admits a Borel unfriendly coloring. Indeed, there is a Borel function $c: X \rightarrow 2$ such that for every $x,|\{y \in N(x): c(x)=c(y)\}| \leqslant 1$.

Proof. Suppose $P_{0}, P_{1}, \ldots$ is the Borel path decomposition of $G$ of length at least 4 . We may assume that this path decomposition satisfies the conclusion of Lemma 3.5.

We inductively construct $c$. At step $i$, we will ensure that every vertex in a path $p \in P_{i}$ has been colored. For all such paths $p \in P_{i}$, inductively, the only vertices in $p$ that can have already been colored must be endpoints of $p$. Hence, there is some extension of our partial coloring so that every vertex of $p$ has at most one adjacent vertex of the same color and the endpoint of $p$ has neighbors of the opposite color. For example, alternate between the two colors along $p$, possibly breaking parity once in the middle of the path. (Here, the reason that paths of length 3 cannot work is if the endpoints of such a path were already assigned opposite colors, one of the endpoints would then gain another vertex of the same color.) Since $P_{i}$ is a path decomposition, each vertex is an interior vertex of at most one path and every edge is contained in some path. Hence, our final coloring $c$ of $X$ has the desired property that each vertex has at most one neighbor of the same color. 
By combining this lemma with Lemma 3.4, we obtain Theorem 1.7 as a corollary.

Suppose $G$ is an acyclic locally finite Borel graph, where every vertex has degree at least 3. An almost identical greedy construction shows that if $G$ has a path decomposition of length at least 3, then $G$ has a Borel perfect matching, and if $G$ has maximum degree $d$, then $G$ has a Borel $d$-list-coloring for any Borel assignment of lists to edges of $G$. For example, this gives us a new way for proving a Baire measurable version of Vizing's theorem for acyclic bounded degree Borel graphs, and the existence of Baire measurable perfect matchings for acyclic locally finite Borel graphs.

\section{Acknowledgements}

The authors would like to thank Anton Bernshteyn, Benson Farb, and Alekos Kechris for helpful discussions about the material of the paper. The authors would also like to thank the anonymous referee for excellent suggestions and thorough reading of the paper.

The first author was partially supported by NSF grants DMS-1500906 and DMS-1855579. The second author was partially supported by NSF grants DMS1500974 and DMS-1764174. The third author was partially supported by NSF grant DMS-1700425.

\section{Conflict of Interest: None.}

\section{References}

[CS] T. Cieśla and M. Sabok, 'Measurable Hall's theorem for actions of abelian groups', Preprint, 2019, arXiv:1903.02987.

[C] D. E. Cohen, Combinatorial Group Theory: a Topological Approach (Cambridge University Press, Cambridge, 1989).

[CK] C. T. Conley and A. S. Kechris, 'Measurable chromatic and independence numbers for ergodic graphs and group actions', Groups Geom. Dyn. 7 (2013), 127-180.

[D] R. Diestel, Graph Theory, Fourth, Graduate Texts in Mathematics, 173 (Springer, Heidelberg, 2010).

[DF] R. Dougherty and M. Foreman, 'Banach-Tarski decompositions using sets with the property of Baire', J. Amer. Math. Soc. 7(1) (1994), 75-124.

[DHK] L. Dubins, M. W. Hirsch and J. Karush, 'Scissor congruence', Israel J. Math. 1 (1963), 239-247.

[GJ] S. Gao and S. Jackson, 'Countable abelian group actions and hyperfinite equivalence relations', Invent. Math. 201(1) (2015), 309-383.

[GJKS] S. Gao, S. Jackson, E. Krohne and B. Seward, 'Forcing constructions and countable Borel equivalence relations', Preprint, 2015. 
[G] R. J. Gardner, 'Convex bodies equidecomposable by locally discrete groups of isometries', Mathematika 32 (1985), 1-9.

[GMP16] L. Grabowski, A. Máthé and O. Pikhurko, 'Measurable equidecompositions for group actions with an expansion property', Preprint, 2016, arXiv:1601.02958.

[GMP17] L. Grabowski, A. Máthé and O. Pikhurko, 'Measurable circle squaring', Ann. of Math (2) 185 (2017), 671-710.

[HM] G. Hjorth and B. D. Miller, 'Ends of graphed equivalence relations, II', Israel J. Math. 169 (2009), 393-415.

[JKL] S. Jackson, A. S. Kechris and A. Louveau, 'Countable Borel equivalence relations', J. Math. Log. 2 (2002), 1-80.

[Ka] S. Katok, Fuchsian Groups, Chicago Lectures in Mathematics (Chicago, 1992).

[K] A. S. Kechris, Classical Descriptive set Theory (Springer, New York, 1995).

[KM] A. S. Kechris and A. S. Marks, 'Descriptive graph combinatorics', Preprint, 2016.

[KST] A. S. Kechris, S. Solecki and S. Todorcevic, 'Borel chromatic numbers', Adv. Math. 141 (1999), 1-44.

[L88] M. Laczkovich, 'Closed sets without measurable matching', Proc. Amer. Math. Soc. 103(3) (1988), 894-896.

[L90] M. Laczkovich, 'Equidecomposability and discrepancy; a solution of Tarski's circlesquaring problem', J. Reine Angew. Math. 404 (1990), 77-117.

[L92] M. Laczkovich, 'Decomposition of sets with small boundary', J. Lond. Math. Soc. 46 (1992), 58-64.

[LN] R. Lyons and F. Nazarov, 'Perfect matchings as IID factors of non-amenable groups', European J. Combin. 32 (2011), 1115-1125.

[MU16] A. Marks and S. Unger, 'Baire measurable paradoxical decompositions via matchings', Adv. Math. 289 (2016), 397-410.

[MU17] A. Marks and S. Unger, 'Borel circle squaring', Ann. of Math. (2) 186 (2017), 581-605.

[Ma] A. Máthé, Measurable Equidecompositions, Proceedings of the International Congress of Mathematicians, 2 (World Sci. Publ., Hackensack, NJ, 2018), 1709-1728.

[Mi] B. D. Miller, 'Ends of graphed equivalence relations, I', Israel J. Math. 169(1) (2009), 375-392.

[N] M. Nadkarni, 'On the existence of a finite invariant measure', Proc. Indian Acad. Sci. Math. Sci. 100 (1990), 203-220.

[SS] S. Schneider and B. Seward, 'Locally nilpotent groups and hyperfinite equivalence relations', Preprint.

[Sr] S. M. Srivastava, A Course on Borel Sets (Springer, New York, 1998).

[Se] J. P. Serre, A Course in Arithmetic, Graduate Texts in Mathematics, 7 (Springer, New York, 1973).

[T] A. Tarski, 'Probléme 38', Fund. Math. 7 (1925), 381.

[W] S. Wagon, The Banach Tarski Paradox (Cambridge University Press, Cambridge, 1986).

[Weh] F. Wehrung, 'Baire paradoxical decompositions need at least six pieces', Proc. Amer. Math. Soc. 121(2) (1994), 643-644. 